\title{
NOTE
}

\section{Abrupt Enlargement of Adrenal Incidentaloma: A Case of Isolated Adrenal Metastasis}

\author{
TOMOKO MIYOSHI, FUMIO OTSUKA, JIRO SUZUKI, KENICHI INAGAKI, YOSHIHIRO KANO, \\ TOSHIO OGURA, KATSUYUKI KIURA*, TAKASHI SAIKA** AND HIROFUMI MAKINO \\ Department of Medicine and Clinical Sciences, Okayama University Graduate School of Medicine, Dentistry and Pharmaceutical \\ Sciences, 2-5-1 Shikata-cho, Okayama 700-8558, Japan \\ *Department of Respiratory Medicine, Okayama University Graduate School of Medicine, Dentistry and Pharmaceutical Sciences, \\ 2-5-1 Shikata-cho, Okayama 700-8558, Japan \\ **Department of Urology, Okayama University Graduate School of Medicine, Dentistry and Pharmaceutical Sciences, 2-5-1 Shikata-cho, \\ Okayama 700-8558, Japan
}

\begin{abstract}
A 56-year-old Japanese man was referred for examination of right adrenal tumor $(3 \mathrm{~cm})$. He had no apparent preexisting cancer by radiological workup and accordingly, the patient was considered as a nonfunctioning adrenocortical adenoma and scheduled for periodic CT scans every 6 months. However, five months after the initial diagnosis the patient complained of severe right back pain with remarkable enlargement of both adrenals ( 20 -fold volume). Although the origin of adrenal tumor was uncertain by pathological workup, positron emission tomography (PET) scan with ${ }^{18} \mathrm{~F}-2$ fluoro-D-deoxyglucose (FDG) eventually revealed a hot spot on left upper lung, which was consistent with a lesion of thickened bulla wall observed by chest CT. The present case is a very rare example of abrupt enlargement of bilateral adrenals due to clinically isolated adrenal metastasis, suggesting the requirement of frequent observation with greatest care regarding morphologic changes of adrenal incidentalomas.
\end{abstract}

Key words: Positron emission tomography, Adrenal incidentaloma, Lung cancer, Adrenal metastasis

(Endocrine Journal 52: 785-788, 2005)

DUE to improvements in radiological diagnosis capabilities, there has been a recent increase in adrenal tumors that are incidentally discovered in patients who underwent medical examinations by ultrasonography (US), computed tomography (CT) and magnetic resonance imaging (MRI), i.e. "adrenal incidentalomas". Diagnostic evaluation of identified adrenal incidentalomas should be performed to determine whether the lesion is hormonally active or nonfunctioning and to determine whether it is malignant or benign [1]. Important hallmarks used as indications for surgical intervention are the size, growth rate and the imaging

Received: April 8, 2005

Accepted: September 7, 2005

Correspondence to: Fumio OTSUKA, M.D., Ph.D., Department of Medicine and Clinical Science, Okayama University Graduate School of Medicine, Dentistry and Pharmaceutical Sciences, 2-5-1 Shikata-cho, Okayama City, 700-8558, Japan characteristics of the tumor as well as the endocrinological behavior. Here, we report a unique case involving an adrenal incidentaloma.

\section{Case Presentation}

A 56-year-old Japanese man was referred to our hospital for examination of right adrenal tumor which was incidentally discovered by abdominal CT. The unilateral tumor ( $3 \mathrm{~cm}$ in diameter) in the right adrenal showed homogeneous CT density (35 to $40 \mathrm{HU}$ ) with capsular enhancement at the early phase (Fig. 1A). On MRI, the tumor did not exhibit any high-intense regions by $\mathrm{T} 2$-weighted image analysis. Basal endocrine data regarding adrenocortical and medullar hormones were as follows: serum cortisol $14.1 \mu \mathrm{g} / \mathrm{dl}$, plasma adrenocorticotropin $30.8 \mathrm{pg} / \mathrm{ml}$, plasma renin activity $1.5 \mathrm{ng} /$ $\mathrm{ml} / \mathrm{h}$, serum aldosterone $109.7 \mathrm{pg} / \mathrm{ml}$, and serum de- 
hydroepiandrosterone sulfate $280 \mu \mathrm{g} / \mathrm{dl}$. Urinary excretions of dopamine, noradrenarine and adrenaline were $665.5,138.6$ and $19.8 \mu \mathrm{g} /$ day, respectively. Serum cortisol level was decreased to $2.9 \mu \mathrm{g} / \mathrm{dl}$ after overnight suppression with $1 \mathrm{mg}$ dexamethasone. The endocrine workup showed that the adrenal tumor was hormonally nonfunctioning. Scintigraphy examination revealed that ${ }^{131}$ I-MIBG did not accumulate in either adrenal, and ${ }^{131}$ I-adosterol exhibited a normal accumulation pattern in both adrenals. Among tumor markers for screening examination slight increase of carcinoembryonic antigen (CEA, $5.89 \mathrm{ng} / \mathrm{ml}$; normal <5) and normal CA19-9 $(8 \mathrm{U} / \mathrm{ml}$; normal $<37)$ were detected. Preexisting cancer was not found in a whole-body checkup, including chest and gastrointestinal examinations performed within a year prior to the diagnosis of the adrenal incidentaloma. Based on these criteria, the incidentaloma was considered as a nonfunctioning adrenocortical adenoma and accordingly the patient was scheduled for periodic CT scans every 6 months in consideration of the possibility of other adrenal malignancies.

Five months after the diagnosis, however, the patient complained of severe right back pain. An emergent CT scan showed that the adrenal glands had enlarged markedly and exhibited heterogenous enhancement $(10 \mathrm{~cm}$ and $4 \mathrm{~cm}$ in right and left adrenals, respectively; Fig. 1B). Abdominal US showed low-echoic adrenals with irregular enlargement (Fig. 1C). Based on these developments in the case a differential diagnosis of adrenocortical cancer, malignant lymphoma, tumor hemorrhage and adrenal metastasis was made. Urinary and serum cortisol were increased, yet the cortisol levels were completely suppressed by $1 \mathrm{mg}$ dexamethasone. ${ }^{67} \mathrm{Ga}$-citrate scintigraphy and ${ }^{99 \mathrm{~m}} \mathrm{Tc}-$ MDP bone scintigraphy showed no particular findings. Whole body CT and endoscopic examination could not detect any primary lesions causing adrenal metastasis. Pathological analysis of surgically resectioned tissue samples revealed that both adrenals exhibited histology consistent with metastatic adenocarcinoma (Fig. 1D) and the same pathological outcome was found in a sample from mesenteric lymph nodes. Regarding tumor markers for adenocarcinoma, levels of CEA $(5.46 \mathrm{ng} / \mathrm{ml})$ and CA19-9 $(6.2 \mathrm{U} / \mathrm{ml})$ were not changed but sialyl Lewis X-i antigen (SLX) was found to be increased $(278.5 \mathrm{U} / \mathrm{ml}$, normal $<38)$. Since the origin of the adrenal tumor was uncertain by pathological workup, positron emission tomography (PET) scan with ${ }^{18} \mathrm{~F}$-2-fluoro-D-deoxyglucose (FDG) was per-
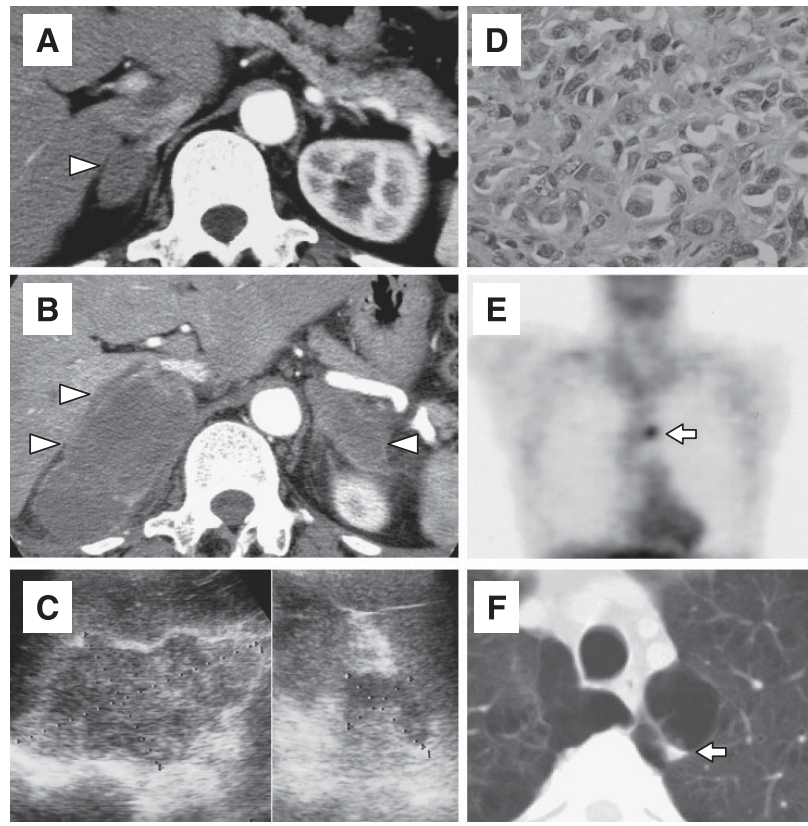

Fig. 1. (A) Right adrenal incidentaloma on the initial CT scan (arrowhead); (B) Bilateral enlargement of adrenal tumors on follow-up CT in 5 months (arrowheads); (C) Ultrasonography of bilateral adrenal tumors; (D) Pathologic feature of the resected adrenal tumor (hematoxylineosin staining, $\times 200$ ); (E) Postoperative FDG-PET scan (arrow indicates a specific accumulation); (F) Chest CT (arrow indicates the corresponding lesion to the spot detected by FDG-PET).

formed after the bilateral adrenalectomy. The FDG tracer specifically accumulated at a spot on left upper lung (Fig. 1E), which was consistent with a lesion of thickened bulla wall observed by chest CT (Fig. 1F).

Upon diagnosis of adrenal metastasis from lung adenocarcinoma, four courses of chemotherapy with paclitaxel, carboplatin and dexamethasone were performed under adequate replacement of hydrocortisone and subsequently gefitinib was also administered. During the course of chemotherapy, serum levels of CEA and SLX transiently decreased but increased again. Primary lesion of lung tumor remained unchanged by chest CT scan at two-year observation, however, peritoneal dissemination with peritonitis carcinomatosa was recently uncovered.

\section{Discussion}

Generally, less than $30 \%$ of incidentalomas increase in size and less than $20 \%$ of those develop biochemical 
abnormalities during 10-year observation [1]. The distributions of the pathologic origins of clinically inapparent adrenal masses vary according to several clinical factors including past cancer history and tumor size [2]. Namely, two thirds of the incidentalomas in populations with no history of cancer are benign tumors, whereas three fourths of adrenal incidentalomas among patients with cancer are metastatic lesions.

The adrenal glands are susceptible organs for metastases from various malignancies. Carcinomas of the lung and breast account for a large proportion of adrenal metastases and other primary lesions include lymphoma, melanoma, leukemia, and kidney and ovarian carcinomas. In a review of autopsy cases, the adrenal glands were involved in $27 \%$ of the cancer cases and the incidence of adrenal metastases in patients with breast and lung cancer is approximately 39 and 35\%, respectively [3]. Among patients with cancer, more than $50 \%$ of clinically inapparent adrenal masses are reported as metastases [4].

Radiologically, the size as well as appearance of adrenal mass is useful for distinguishing benign from malignant tumors. Available data regarding the size suggest that lesions smaller than $4 \mathrm{~cm}$ are generally benign [1]. The CT attenuation value which is conventionally expressed as Hounsfield units (HU) may enable differentiation between benign and malignant lesions [5]. A homogeneous mass with smooth border and low attenuation value less than $10 \mathrm{HU}$ on unenhanced CT strongly suggests a benign adrenal adenoma $[6,7]$. However, benign adenomas are not always characterized by unenhanced CT since lipidpoor adenomas can be occasionally discovered $[8,9]$. In such cases, the presence of rapid washout of enhancement is useful to distinguish the benign adenomas from metastasis. Our case showed relatively high attenuation values (35 to $40 \mathrm{HU}$ ) suggesting non-adenomatous characteristics, while the tumor also showed homogenous density with smooth border and rapid washout by enhanced $\mathrm{CT}$.

MRI is known to be effective in distinguishing benign from malignant lesions. Metastases are usually hypointense on T1-weighted images but hyperintense on T2-weighted images [2]. In particular, benign adenomas exhibit clear suppression of the signals on chemical-shift imaging [10]. Unfortunately, chemicalshift MRI was not performed at the initial examination in our present case. The fat suppression images would have been helpful to distinguish between adenoma and metastasis, although it may not provide additional information beyond that obtained by unenhanced CT scan [1].

In patients with nonfunctioning adrenal tumors, differential diagnosis between malignant and benign tumors is critical for the subsequent management. Variables to be considered include not only tumor size and imaging features, but also its growth rate. In cases of incidentalomas greater than $6 \mathrm{~cm}$, the tumor is highly likely malignant and therefore surgery should be readily considered [1]. In contrast, the optimal diagnostic evaluation has not been well established for smaller adrenal masses such as 4 to $6 \mathrm{~cm}$ in size. If these lesions are hormonally inactive and exhibit benign imaging appearances, most of the tumors will be simply monitored. The present case made us realize the risk of abrupt enlargement ( $\sim 20$-fold volume in 5 months) of clinically isolated adrenal metastasis [11] in the course of tumor monitoring. As for the management of adrenal metastasis, adrenalectomy has no known benefits for the patients who suffer adrenal metastases from known primary lesions. Nevertheless, surgical removal of the isolated adrenal metastasis with unknown origin is worthy of consideration to achieve prolonged survival [12].

This case also demonstrates the benefits of FDGPET to uncover origins of clinically inapparent adrenal metastasis. FDG-PET scan is a highly sensitive tool for staging of lung cancer and in particular, detecting its mediastinal lesions [13]. Advantages of FDG-PET have been suggested for the characterization of adrenal masses in patients with either clinically inapparent adrenal masses or cancer work-up including differentiation of malignant from benign adrenal masses [14, 15]. It may be a useful tool as initial examination for detecting preexisting cancer for patients with adrenal incidentalomas that lack typical radiologic signs of adrenocortical adenoma.

Given that prevalence of adrenal incidentaloma increases with age, our case suggests a requirement for more frequent follow-up with greatest care to detect morphologic changes of adrenal tumors or, if possible, direct pathological approaches especially when the key characteristics of benign adenomas were not completely matched. Careful consideration of the clinical condition in individual patients is necessary when making treatment decision. Valid hallmarks to characterize adrenal incidentalomas are urgently needed in order to diagnose their pathological features non-invasively. 


\section{References}

1. Grumbach MM, Biller BM, Braunstein GD, Campbell KK, Carney JA, Godley PA, Harris EL, Lee JK, Oertel YC, Posner MC, Schlechte JA, Wieand HS (2003) Management of the clinically inapparent adrenal mass ("incidentaloma"). Ann Intern Med 138: 424-429.

2. Mansmann G, Lau J, Balk E, Rothberg M, Miyachi Y, Bornstein SR (2004) The clinically inapparent adrenal mass: update in diagnosis and management. Endocr Rev 25: 309-340.

3. Abrams HL, Spiro R, Goldstein N (1950) Metastases in carcinoma; analysis of 1000 autopsied cases. Cancer 3 : 74-85.

4. Lenert JT, Barnett CC Jr, Kudelka AP, Sellin RV, Gagel RF, Prieto VG, Skibber JM, Ross MI, Pisters PW, Curley SA, Evans DB, Lee JE (2001) Evaluation and surgical resection of adrenal masses in patients with a history of extra-adrenal malignancy. Surgery 130: 1060-1067.

5. Lee MJ, Hahn PF, Papanicolaou N, Egglin TK, Saini S, Mueller PR, Simeone JF (1991) Benign and malignant adrenal masses: CT distinction with attenuation coefficients, size, and observer analysis. Radiology 179: 415-418.

6. Korobkin M, Brodeur FJ, Yutzy GG, Francis IR, Quint LE, Dunnick NR, Kazerooni EA (1996) Differentiation of adrenal adenomas from nonadenomas using CT attenuation values. AJR Am J Roentgenol 166: 531-536.

7. Szolar DH, Kammerhuber F (1997) Quantitative CT evaluation of adrenal gland masses: a step forward in the differentiation between adenomas and nonadenomas? Radiology 202: 517-521.

8. Pena CS, Boland GW, Hahn PF, Lee MJ, Mueller PR (2000) Characterization of indeterminate (lipid-poor) adrenal masses: use of washout characteristics at contrast-enhanced CT. Radiology 217: 798-802.

9. Kebapci M, Kaya T, Gurbuz E, Adapinar B, Kebapci N, Demirustu C (2003) Differentiation of adrenal adenomas (lipid rich and lipid poor) from nonadenomas by use of washout characteristics on delayed enhanced CT. Abdom Imaging 28: 709-715.

10. Outwater EK, Siegelman ES, Huang AB, Birnbaum BA (1996) Adrenal masses: correlation between CT attenuation value and chemical shift ratio at MR imaging with in-phase and opposed-phase sequences. Radiology 200: 749-752.

11. Lee JE, Evans DB, Hickey RC, Sherman SI, Gagel RF, Abbruzzese MC, Abbruzzese JL (1998) Unknown primary cancer presenting as an adrenal mass: frequency and implications for diagnostic evaluation of adrenal incidentalomas. Surgery 124: 1115-1122.

12. Kim SH, Brennan MF, Russo P, Burt ME, Coit DG (1998) The role of surgery in the treatment of clinically isolated adrenal metastasis. Cancer 82: 389-394.

13. Coleman RE (2001) PET in lung cancer staging. $Q J$ Nucl Med 45: 231-234.

14. Boland GW, Goldberg MA, Lee MJ, Mayo-Smith WW, Dixon J, McNicholas MM, Mueller PR (1995) Indeterminate adrenal mass in patients with cancer: evaluation at PET with 2-[F-18]-fluoro-2-deoxy-Dglucose. Radiology 194: 131-134.

15. Maurea S, Mainolfi C, Bazzicalupo L, Panico MR, Imparato $\mathrm{C}$, Alfano $\mathrm{B}$, Ziviello $\mathrm{M}$, Salvatore $\mathrm{M}$ (1999) Imaging of adrenal tumors using FDG PET: comparison of benign and malignant lesions. AJR Am J Roentgenol 173: 25-29. 